\title{
Congenital amegakaryocytic thrombocytopenia
}

INSERM

\section{Source}

INSERM. (1999). Orphanet: an online rare disease and orphan drug data base. Congenital amegakaryocytic thrombocytopenia. ORPHA:3319

Congenital amegakaryocytic thrombocytopenia (CAMT) is a rare inherited bone marrow failure syndrome characterized by an isolated and severe decrease in the number of platelets and megakaryocytes during the first years of life that develops into bone marrow failure with pancytopenia later in childhood. 